\title{
Sperm DNA fragmentation in cryopreserved samples from subjects with different cancers
}

\author{
Lara Tamburrino $^{\mathrm{A}}$, Marta Cambi ${ }^{\mathrm{A}}$, Sara Marchiani ${ }^{\mathrm{A}}$, Ilaria Manigrasso ${ }^{\mathrm{A}}$, \\ Selene Degl'Innocenti ${ }^{\mathrm{B}}$, Gianni Forti ${ }^{\mathrm{A}}$, Mario Maggi ${ }^{\mathrm{A}}$, \\ Elisabetta Baldi ${ }^{\mathrm{A}, \mathrm{C}}$ and Monica Muratori ${ }^{\mathrm{A}, \mathrm{C}}$ \\ A Department of Biomedical, Experimental and Clinical Sciences, Centre of Excellence DeNothe, \\ University of Florence, Viale Pieraccini 6, I-50139 Florence, Italy. \\ ${ }^{B}$ Azienda Ospedaliera-Universitaria Careggi, Florence, Viale Pieraccini 6, I-50139 Florence, Italy. \\ ${ }^{\mathrm{C}}$ Corresponding authors. Emails: elisabetta.baldi@unifi.it; monica.muratori@unifi.it
}

\begin{abstract}
Sperm cryopreservation is widely used by cancer patients undergoing chemo- or radiotherapy. Evidence suggests that IVF outcome with cryopreserved spermatozoa from cancer patients is less successful. To determine whether sperm DNA fragmentation (SDF) is involved in the lower fertilising ability of cryopreserved spermatozoa of cancer patients, SDF was evaluated in thawed spermatozoa from 78 men affected by different cancers and 53 men with noncancer pathologies. SDF was assessed by the terminal deoxyribonucleotidyl transferase-mediated dUTP-digoxigenin nick end-labelling (TUNEL), propidium iodide (PI), flow cytometry procedure, which allows determination of two different cell populations ( $\mathrm{PI}^{\text {brighter }}$ and $\mathrm{PI}^{\text {dimmer }}$ ) and thus to determine the percentage of DNA fragmented sperm in both. PI ${ }^{\text {dimmer }}$ spermatozoa are totally unviable, whereas PI $^{\text {brighter }}$ spermatozoa with SDF may be motile and morphologically normal, having higher biological relevance in the reproductive process. We found that the proportion of DNA fragmented PI ${ }^{\text {brighter }}$ cells was significantly higher in thawed spermatozoa from cancer than non-cancer patients. Moreover, a positive correlation was found between the degree of DNA fragmentation and sperm motility in the PIbrighter population of spermatozoa from cancer patients that wasn't seen in non-cancer patients. The results of the present study suggest that higher SDF levels may contribute to the lower IVF success of cryopreserved spermatozoa from cancer patients and that evaluation of SDF could complement genetic counselling as part of the routine management of cancer patients who seek fertility preservation.
\end{abstract}

Additional keywords: cancer patients, flow cytometry, propidium iodide, terminal deoxyribonucleotidyl transferasemediated dUTP-digoxigenin nick end-labelling (TUNEL).

Received 18 March 2015, accepted 14 September 2015, published online 14 October 2015

\section{Introduction}

Sperm cryopreservation is currently the only feasible option for preserving the future fertility of male cancer patients undergoing chemotherapy, radiotherapy or any other treatment potentially detrimental to testicular function. Despite extensive sperm banking, the degree to which cryopreserved semen is used in ART remains low, approaching only 10\% (van Casteren et al. 2008; Bizet et al. 2012; Botchan et al. 2013; Ping et al. 2014). Consequently, studies on ART outcome using cryopreserved semen from cancer patients suffer from possible bias because of this low rate, with variable results reported (see table 1 in van Casteren et al. 2008). However, the available studies indicate that a lower pregnancy rate is achieved after IVF cycles using cryopreserved spermatozoa of cancer patients (averaging 18\%; see table 1 of van Casteren et al. 2008) compared with cryopreserved spermatozoa from healthy donors (averaging 39\%; Clarke et al. 1997; Tomlinson et al. 2010; Nordqvist et al. 2014).
These data have been substantiated by a recent study reporting that IVF with cryopreserved spermatozoa from cancer patients fails to produce pregnancies because of a lack of oocyte fertilisation or implantation (Botchan et al. 2013). The reasons for lower IVF success with cryopreserved spermatozoa from cancer patients are currently unknown. One reason could be the low semen quality of cancer patients. However, although low semen quality has often been reported at the time of cryopreservation of spermatozoa from men with testicular cancer (Said et al. 2009; Botchan et al. 2013; Degl'Innocenti et al. 2013), it remains less clear whether semen quality is affected in the case of other types of cancer (e.g. leukaemia and Hodgkin's and non-Hodgkin's lymphoma; Hallak et al. 1999; Rofeim and Gilbert 2004; Smit et al. 2010; Degl'Innocenti et al. 2013; Bujan et al. 2014). Further, for haematological or other types of cancers, the recovery of sperm motility and/or viability following cryopreservation has been shown to be similar to that of non-cancer 
patients (Degl'Innocenti et al. 2013). Of the type of sperm damage that may affect IVF success, sperm DNA fragmentation (SDF; Zribi et al. 2010; Ribas-Maynou et al. 2014) appears to be of considerable importance for reproductive outcome. Indeed, SDF is associated with reduced ART outcomes (e.g. embryo quality, fertilisation rate, cleavage rate) (for a review, see Tamburrino et al. 2012) and an increased risk of miscarriages (Zini et al. 2008; Robinson et al. 2012). Interestingly, Meseguer et al. (2008) found similar post-thawing SDF levels in cancer patients and men whose partners failed to achieve pregnancy in IVF cycles, which suggests a role for DNA damage in the reduced IVF results of cancer patients (Botchan et al. 2013). However, whether cancer is per se associated with increased SDF remains contentious. For example, in the case of testicular cancer, high levels of SDF have been reported in some studies (O'Flaherty et al. 2008; Meseguer et al. 2008), but not in others (Ribeiro et al. 2008; Ståhl et al. 2009; Smit et al. 2010). Similarly, elevated SDF levels have been found in samples from men with non-Hodgkin's lymphoma by some (Meseguer et al. 2008; Smit et al. 2010), but not by others (O'Flaherty et al. 2008; McDowell et al. 2013). Whether there are different levels of SDF following the cryopreservation of spermatozoa from men with cancer than from men with non-cancer pathologies is even less clear, with only two studies (Edelstein et al. 2008; Meseguer et al. 2008) evaluating post-cryopreservation SDF levels in cancer patients. Although both studies reported higher SDF levels in cancer patients compared with donors (Edelstein et al. 2008; Meseguer et al. 2008), SDF was evaluated in a small number (hundreds) of spermatozoa and determined either microscopically after the terminal deoxyribonucleotidyl transferase-mediated dUTP-digoxigenin nick end-labelling (TUNEL) assay (Edelstein et al. 2008) or by using the sperm chromatin dispersion test (SCDt; Meseguer et al. 2008). There is evidence that evaluating the number of TUNEL-positive cells under a microscope underestimates the percentage of spermatozoa with SDF in a given sample (Domínguez-Fandos et al. 2007; Muratori et al. 2008a). Conversely, the SCDt, which requires an initial denaturation step, evaluates 'potential' DNA damage rather than the actual single- or double-strand DNA breaks as evaluated by the TUNEL assay (Henkel et al. 2010).

Recently, we developed a new cytofluorimetric method to evaluate SDF, namely the TUNEL-propidium iodide (PI) method (Muratori et al. 2008b, 2010). Using this method, we demonstrated the existence of two cytometric sperm populations, namely $\mathrm{PI}^{\text {brighter }}$ and $\mathrm{PI}^{\text {dimmer }}$, so called because of the different intensity of nuclear staining, which exhibit different viability: $\mathrm{PI}^{\text {dimmer }}$ spermatozoa are all dead, whereas $\mathrm{PI}^{\text {brighter }}$ spermatozoa are partly dead (Marchiani et al. 2011). The percentage of spermatozoa with DNA fragmentation in the two populations differs, with the percentage of SDF in the $\mathrm{PI}^{\text {brighter }}$ population being variable but the $\mathrm{PI}^{\text {dimmer }}$ population consisting of $100 \%$ spermatozoa with DNA fragmentation (Muratori et al. 2008b). In addition, a fraction of the PI ${ }^{\text {dimmer }}$ population consists of spermatozoa with a large loss of chromatin material (Marchiani et al. 2014a). Most importantly, we have shown that although there is a negative correlation between the $\mathrm{PI}^{\text {dimmer }}$ population and semen parameters, the percentage of spermatozoa with SDF in the PI ${ }^{\text {brighter }}$ population is unrelated to semen quality (Muratori et al. 2008b). Thus, although there is no chance that $\mathrm{PI}^{\text {dimmer }}$ spermatozoa will be used in IVF or intracytoplasmic sperm injection (ICSI), DNA-fragmented $\mathrm{PI}^{\text {brighter }}$ spermatozoa may be motile and/or morphologically normal, and so have some probability of being used to fertilise oocytes, thus affecting reproductive outcome. The TUNEL-PI procedure enables the determination of SDF in a large number of cells and to focus on the DNA fragmented sperm population, which is likely to affect reproduction.

In the present study, using the TUNEL-PI procedure, we evaluated SDF in cryopreserved spermatozoa from cancer patients (testicular cancer and Hodgkin's and non-Hodgkin's lymphoma) and compared the results with those of patients cryopreserving spermatozoa because of autoimmune pathologies or subfertility. The aim of the study was to determine whether the extent of sperm DNA damage contributed to the lower fertilisation ability of thawed spermatozoa from cancer patients.

\section{Materials and methods \\ Chemicals}

Test yolk cryopreservation medium was purchased from Irvine Scientific (Santa Ana, CA, USA). Human tubal fluid (HTF) was purchased from Celbio (Milan, Italy). The In Situ Cell Death Detection Kit, Fluorescein was purchased from Roche Molecular Biochemicals (Milan, Italy). PI was obtained from Calbiochem (Nottingham, UK). Sperm Vitalstain was obtained from Nidacon (Molndal, Sweden). All other reagents were obtained from Sigma Aldrich (Milan, Italy).

\section{Patients}

The study was conducted using semen collected from 131 patients undergoing semen cryopreservation in the Laboratory of Andrology of the Azienda Ospedaliera-Universitaria of Careggi (Florence, Italy) between 2000 and 2012 who discontinued sperm banking. In order to enable evaluation of SDF, only cryopreserved semen samples with at least 1 million spermatozoa per ejaculate were included in the study. Of the 131 samples, 42 were cryopreserved because of lymphoma (22 Hodgkin's lymphoma; 20 non-Hodgkin's lymphoma), 36 were cryopreserved because of testicular cancer (19 seminoma; 17 non-seminomatous germ cell tumours) and 53 were cryopreserved because of non-cancer pathologies (21 autoimmune pathologies (15 multiple sclerosis, 6 other autoimmune pathologies), 32 subfertility). None of the patients had Type 1 or 2 diabetes. Spermatozoa from all cancer patients were cryopreserved before the initiation of any antineoplastic chemotherapy or radiotherapy. In the case of testicular cancer patients, samples were cryopreserved after orchiectomy. In the case of subfertile men, samples were cryopreserved to ensure spermatozoa were available for subsequent IVF or because a decline in sperm production with time was suspected. In the case of men suffering from autoimmune pathologies, samples were cryopreserved because the treatments they were undergoing were potentially toxic to the gonads. All data were collected as part of routine clinical procedures and therefore, according to the Italian law, approval from the local Ethics Committee was not 
required. Informed consent was obtained from all patients to use discarded, cryopreserved spermatozoa for research purposes.

\section{Pre- and post-treatment semen analysis}

Semen analysis was performed according to World Health Organization (WHO) guidelines (WHO 1999). Sperm count was evaluated only before cryopreservation, whereas motility and viability were evaluated both before and after cryopreservation. The percentage of progressive, non-progressive and immotile spermatozoa was determined for 200 spermatozoa per sample. Post-cryopreservation sperm viability was evaluated using an eosin test (WHO 1999). The Laboratory of Andrology (Azienda Ospedaliera-Universitaria of Careggi) has been part of the UK National External Quality Assessment Service (NEQAS) program for semen analysis since 2005 . The mean $( \pm$ s.d.) percentage bias of the Laboratory for 2013-14 for progressive motility and sperm concentration was $9.2 \pm 4.7 \%$ and $8.7 \pm 4.1 \%$, respectively ( $n=7$; data from UK NEQAS; Degl'Innocenti et al. 2013). Sperm morphology data were not analysed. The methods used to assess sperm morphology varied during the study years; until January 2008, the fourth edition of the WHO Laboratory Manual for the Examination and Processing of Human Semen was used (WHO 1999), whereas after January 2008 we used the fifth edition of the manual (WHO 2010). Thus, the sperm morphology data did not lend themselves to the large-scale data analysis needed for the present study.

\section{Cryopreservation of semen samples}

Semen samples were collected on the same day as cryopreservation by masturbation in the laboratory. In few exceptional cases, semen collection was performed at home. All subjects were asked to observe 2-7 days of sexual abstinence prior to sample collection. After semen analysis, semen samples were frozen in liquid nitrogen tanks using a manually controlled freezing procedure as described by Gandini et al. (2006) with minor modifications. Briefly, samples were diluted $1: 1(\mathrm{v} / \mathrm{v})$ by drop-wise addition of test yolk buffer with glycerol and gentamicin (Irvine Scientific, Santa Ana, CA, USA). After equilibration at room temperature for 5-10 $\mathrm{min}$, spermatozoa were loaded in 500- $\mu$ L high-security sperm straws (Cryo Bio System, Ouen Sur Iton, France). Straws were frozen by exposing them for $8 \mathrm{~min}$ to liquid nitrogen vapour before plunging them into liquid nitrogen. Straws were thawed by transferring them to room temperature for $15 \mathrm{~min}$, followed by $15 \mathrm{~min}$ at $37^{\circ} \mathrm{C}$; semen samples were then evaluated.

\section{Evaluation of SDF}

SDF was evaluated using the TUNEL-PI assay (Muratori et al. 2008b). After thawing, semen samples were washed twice with HTF medium to completely remove seminal plasma and cryopreservation extender. Next, spermatozoa were fixed in $4 \%$ paraformaldehyde (PFA) and immediately processed for TUNEL labelling. Spermatozoa were centrifuged at $500 \mathrm{~g}$ for $10 \mathrm{~min}$ at room temperature and then washed twice with $200 \mu \mathrm{L}$ phosphate-buffered saline (PBS) with $1 \%$ bovine serum albumin (BSA). The spermatozoa were then permeabilised with $0.1 \%$ Triton $\mathrm{X}-100$ in $100 \mathrm{~mL}$ of $0.1 \%$ sodium citrate for $4 \mathrm{~min}$ on ice. After two washes with $200 \mu \mathrm{L}$ PBS-BSA $1 \%$, spermatozoa were incubated in $50 \mu \mathrm{L}$ labelling solution (supplied with the In Situ Cell Death Detection Kit, Fluorescein) containing terminal deoxyribonucleotidyl transferase (TdT) for $1 \mathrm{~h}$ at $37^{\circ} \mathrm{C}$ in the dark. Finally, samples were washed twice with $200 \mu \mathrm{L}$ PBS-BSA $1 \%$, resuspended in $500 \mu \mathrm{L}$ PBS, stained with $10 \mu \mathrm{L}$ PI ( $30 \mu \mathrm{g} \mathrm{mL}^{-1}$ in PBS), and incubated in the dark for $15 \mathrm{~min}$ at room temperature. For each test sample, a negative control (omitting TdT) and a sample for fluorescence compensation (labelled only with TUNEL) were prepared. For each sample, 8000 events were recorded within the flame-shaped region (R1) characteristic of spermatozoa (Muratori et al. 2008b) in the forward light scatter (FSC)/side light scatter (SSC) dot plot (FSC detector: voltage set E00, AmpGain 6.47, linear scale, threshold 68; SSC detector: voltage set 396, AmpGain 1, linear scale). After gating (R2) the nucleated events (i.e. the events labelled with PI), SDF was determined in the intersection between R1 and R2. This strategy guarantees that fluorescence is analysed in a population formed only by spermatozoa (Muratori et al. 2008b; Marchiani et al. 2014a), excluding debris, large cells and semen apoptotic bodies (Marchiani et al. 2007). In the present study we did not use any tool for discrimination of doublets; however, these rarely occur in suspensions of spermatozoa at the concentration used herein. Indeed, we have shown that the that count of spermatozoa by flow cytometry strictly overlaps that obtained using light microscopy (Marchiani et al. 2014a). Green fluorescence (of nucleotide conjugated with fluorescein) was revealed by the FL- 1 detector $(515-555 \mathrm{~nm}$ wavelength band, voltage set 590) of a FACScan flow cytometer (Becton Dickinson, Mountain View, CA, USA). To reveal red fluorescence (of PI), we used the FL-2 detector (563-607 nm wavelength band, voltage set 477), which, unlike FL-3, requires fluorescence compensation (see below). Flow cytometry data were acquired and analysed by Cell Quest software (Becton Dickinson) and stored as list mode data in standard fcs format. For analysis of flow cytometry data, in each of the two sperm populations (brighter and dimmer; Fig. 1), a vertical marker was established in the TUNEL axis of the dot plot of negative control (TdT omitted), including 99\% of total events. This marker was translated to the corresponding test sample and all the events beyond the marker were considered TUNEL positive. Discrimination between $\mathrm{PI}^{\text {dimmer }}$ and $\mathrm{PI}^{\text {brighter }}$ sperm populations was established by a horizontal marker in the PI axis (Fig. 1). 'PI ${ }^{\text {dimmer }} \mathrm{SDF}$ ' corresponds to $\mathrm{PI}^{\text {dimmer }}$ spermatozoa as a percentage of the total sperm population, because PI ${ }^{\text {dimmer }}$ spermatozoa are $100 \%$ DNA fragmented (see Fig. 1). Total SDF was calculated by adding $\mathrm{PI}^{\text {brighter }} \mathrm{SDF}$ and $\mathrm{PI}^{\text {dimmer }} \mathrm{SDF}$ (Muratori et al. 2008b).

\section{Statistical analysis}

Statistical analysis was performed using SPSS version 20.0 for Windows (IBM SPSS Statistics for Windows, Armonk, NY, USA). The Kolmogorov-Smirnov test was used to test the normality of data distribution. Data are expressed as the mean \pm s.d. when normally distributed (age, pre-cryopreservation total and progressive motility) and as median (range) when not normally distributed (sperm number, post-cryopreservation total and progressive motility, pre- and post-cryopreservation 

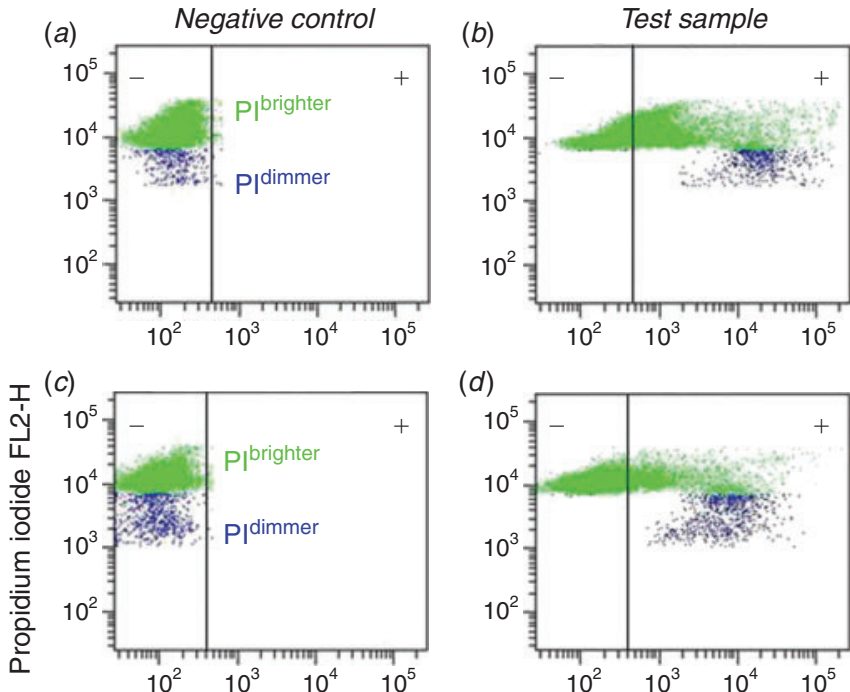

(d)
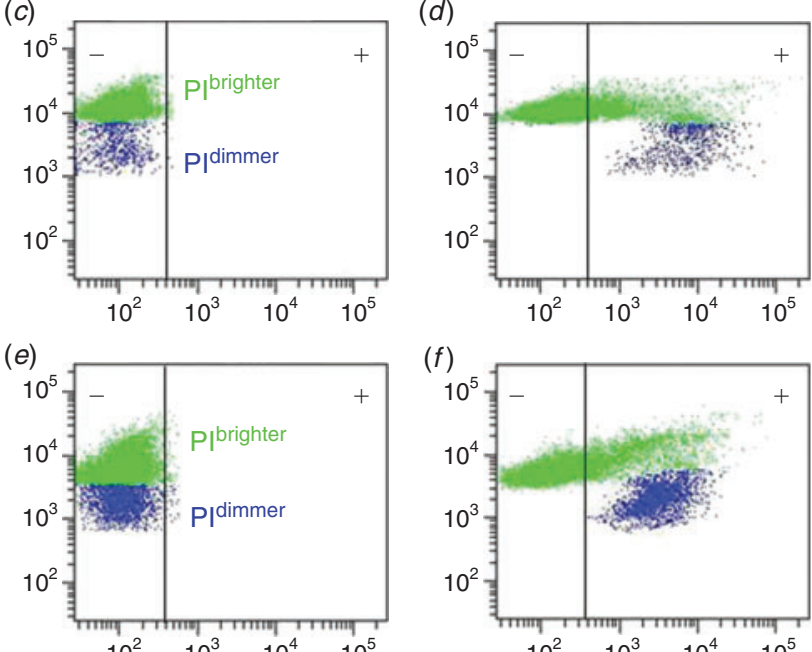

$(f)$

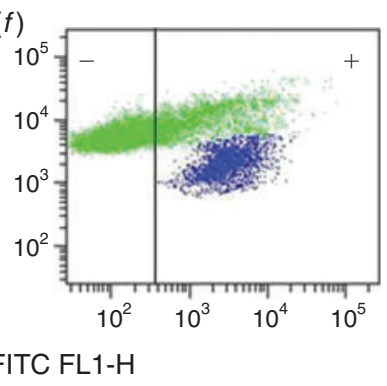

Fig. 1. Typical terminal deoxyribonucleotidyl transferase-mediated dUTP-digoxigenin nick end-labelling (TUNEL)-propidium iodide (PI) dot plots of negative controls and corresponding test samples for $(a, b)$ a patient with non-seminomatous germ cell tumours, $(c, d)$ a patient with autoimmune pathologies and $(e, f)$ a subfertile patient. Flow cytometry distinguished two cytometric populations differing in the intensity of nuclear staining ( $\mathrm{PI}^{\text {brighter }}$ (green) and $\mathrm{PI}^{\text {dimmer }}$ (blue)). The vertical lines show the demarcation between TUNEL-positive and -negative cells. Note that all $\mathrm{PI}^{\mathrm{dimmer}}$ spermatozoa are within the TUNEL-positive quadrant.

viability, and $\mathrm{PI}^{\text {brighter }}$, total and $\mathrm{PI}^{\text {dimmer }} \mathrm{SDF}$ ). In the case of non-normally distributed parameters, the significance of differences between groups was evaluated by the Kruskal-Wallis and post hoc Mann-Whitney non-parametric tests. In the case of normally distributed parameters, one-way analysis of variance (ANOVA) and unpaired two-sided Student's $t$-test were used. $P<0.05$ was considered significant. Correlations were assessed by Spearman's correlation tests. Multiple linear regression analysis was applied when indicated.

\section{Results}

Pre- and post-cryopreservation sperm parameters

Pre- and post-cryopreservation motility, viability and number of spermatozoa, as well as the age of the patients at the time of cryopreservation, are given in Table 1. Compared with autoimmune pathologies, all cancer groups, except for the Hodgkin's lymphoma group, had significantly lower numbers of spermatozoa at the time of cryopreservation, whereas

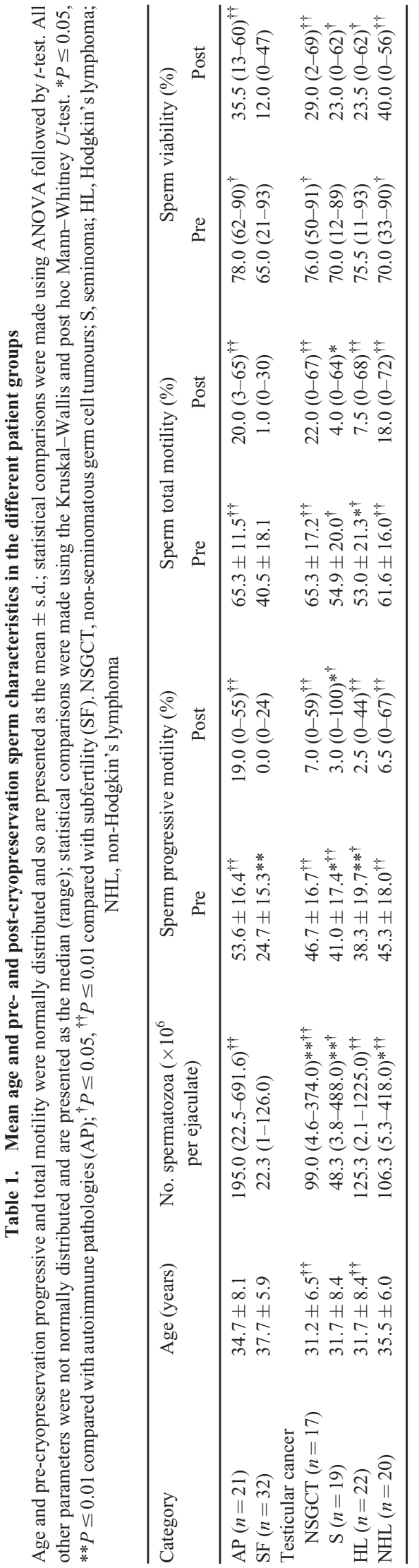



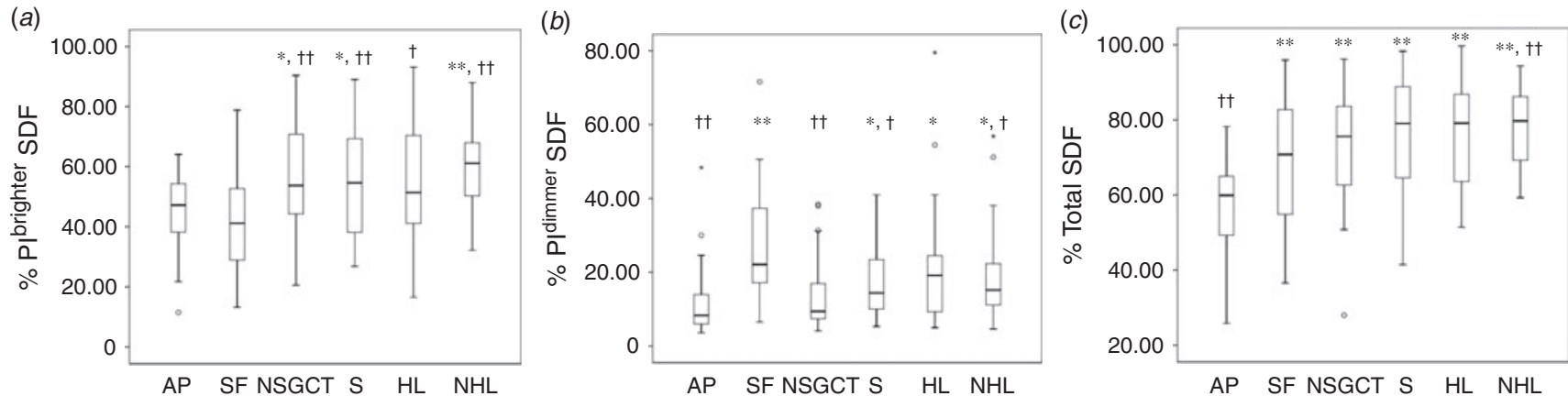

Fig. 2. Sperm DNA fragmentation in cryopreserved samples from patients with different types of cancer, subfertility and autoimmune pathologies. Data show the percentage of spermatozoa with DNA fragmentation within $(a) \mathrm{PI}^{\text {brighter }}(b) \mathrm{PI}^{\text {dimmer }}$ and (c) total sperm populations. Within the boxes, the horizontal line indicates the median, the boxes represent the interquartile range, the whiskers indicate minimum and maximum values and the circles represent outliers. ${ }^{*} P \leq 0.05, * * P \leq 0.01$ compared with autoimmune pathologies (AP; $n=21$ ); ${ }^{\dagger} P \leq 0.05$, ${ }^{\dagger \dagger} P \leq 0.01$ compared with subfertility (SF; $n=32)$. NSGCT, non-seminomatous germ cell tumours $(n=17)$; S, seminoma $(n=19)$; HL, Hodgkin's lymphoma $(n=22)$; NHL, non-Hodgkin's lymphoma $(n=20)$.

Table 2. Correlations between sperm DNA fragmentation (SDF) and post-thaw sperm motility in cancer and non-cancer subjects, as derived from univariate Spearman's regression analysis

\begin{tabular}{|c|c|c|c|c|c|c|}
\hline & \multicolumn{2}{|c|}{ Post-thaw progressive sperm motility (\%) } & \multicolumn{2}{|c|}{ Post-thaw total sperm motility (\%) } & \multicolumn{2}{|c|}{ Post-thaw sperm viability (\%) } \\
\hline & Cancer $(n=78)$ & Non-cancer $(n=53)$ & Cancer $(n=78)$ & Non-cancer $(n=53)$ & Cancer $(n=78)$ & Non-cancer $(n=53)$ \\
\hline $\mathrm{PI}^{\text {brighter }} \mathrm{SDF}$ & $r=0.4, P<0.001$ & $r=0.1$, n.s. & $r=0.5, P<0.001$ & $r=0.1, \mathrm{n} . \mathrm{s}$ & $r=0.4, P<0.001$ & $r=0.1$, n.s. \\
\hline $\mathrm{PI}^{\text {dimmer }} \mathrm{SDF}$ & $r=-0.5, P<0.001$ & $r=-0.5, P<0.001$ & $r=-0.6, P<0.001$ & $r=-0.4, P<0.01$ & $r=-0.6, P<0.001$ & $r=-0.3, P<0.05$ \\
\hline Total SDF & $r=0.03$, n.s. & $r=-0.3, P<0.05$ & $r=-0.02$, n.s. & $r=-0.2$, n.s. & $r=-0.4$, n.s. & $r=-0.2$, n.s. \\
\hline
\end{tabular}

progressive and total motility were lower for samples from patients with seminoma and Hodgkin's lymphoma (Table 1). Compared with subfertile men, patients from all cancer groups had higher numbers of spermatozoa and progressive and total motility at the time of cryopreservation. In agreement with previous results (Degl'Innocenti et al. 2013), the lowest motility and viability after thawing was observed in subfertile, Hodgkin's lymphoma and seminoma patients (Table 1). The mean age of patients was similar in all cancer groups. Patients with nonseminomatous germ cell tumours and Hodgkin's lymphoma were, on average, significantly younger than subfertile patients (Table 1). When the age of all cancer patients was compared with that of non-cancer patients, the former group was found to be younger ( $33.3 \pm 7.1$ vs $36.5 \pm 6.9$ years; $P<0.05$ ).

\section{Post-cryopreservation SDF}

Fig. 1 shows typical cytograms of TUNEL-PI post-thaw sperm samples from a patients affected by cancer (Fig. $1 a, b$ ), autoimmune pathology (Fig. 1c,d) or subfertility (Fig. 1e, f). PI staining was able to distinguish the two sperm populations, namely the $\mathrm{PI}^{\text {brighter }}$ (green) and PI ${ }^{\text {dimmer }}$ (blue) populations. The percentage of TUNEL-positive spermatozoa within each of the two populations (Fig. $1 b, d, f$ ) can be calculated after setting a marker on the TUNEL-negative control (Fig. $1 a, c, e$ ). Note that this value is $100 \%$ for the $\mathrm{PI}^{\mathrm{dimmer}}$ population (Fig. $1 b, d, f$; Muratori et al. 2008b).
Fig. 2 shows $\mathrm{PI}^{\text {brighter }}$ (Fig. 2a), $\mathrm{PI}^{\text {dimmer }}$ (Fig. $2 b$ ) and total (Fig. 2c) SDF in the different groups of patients. PI ${ }^{\text {brighter }}$ SDF was significantly higher in patients with non-seminomatous germ cell tumours, seminoma and non-Hodgkin's lymphoma compared with patients in both the subfertile and autoimmune pathology groups. Patients with Hodgkin's lymphoma had significantly higher $\mathrm{PI}^{\text {brighter }}$ SDF compared with subfertile men, and a tendency for higher $\mathrm{PI}^{\text {brighter }} \operatorname{SDF}(P=0.07)$ compared with patients with autoimmune pathology, although the difference did not reach statistical significance. PI ${ }^{\text {dimmer }}$ SDF was significantly higher in subfertile patients compared with all other patient groups, except for those with Hodgkin's lymphoma. Patients affected by lymphomas and seminoma had higher $\mathrm{PI}^{\text {dimmer }}$ SDF than men with autoimmune pathologies. Total SDF was significantly higher in all cancer groups compared with patients with autoimmune pathologies. No differences in $\mathrm{PI}^{\text {brighter }}, \mathrm{PI}^{\text {dimmer }}$ and total SDF were observed among the different cancer groups.

Correlations between SDF and pre- and postcryopreservation sperm parameters

Correlations between SDF levels in $\mathrm{PI}^{\text {brighter }}$ and $\mathrm{PI}^{\text {dimmer }}$ populations and pre- and post-cryopreservation sperm motility and viability in cancer and non-cancer patients are given in Table 2. There was no significant relationship between PI ${ }^{\text {brighter }}$ SDF and pre- or post-cryopreservation motility and viability in 
non-cancer patients. In cancer patients, $\mathrm{PI}^{\text {brighter }}$ SDF was positively correlated with pre- and post-cryopreservation motility and viability (Table 2). To determine whether such relationships were affected by patient age (a factor established to affect SDF; Moskovtsev et al. 2006; Wyrobek et al. 2006; Schmid et al. 2007) and the type of cancer, multiple regression analysis was performed including these two variables. All positive correlations between $\mathrm{PI}^{\text {brighter }} \mathrm{SDF}$ and pre- and post-cryopreservation progressive motility (adjusted $r=0.47(P<0.001)$ and $r=0.35$ $(P<0.005)$, respectively), pre- and post-cryopreservation total motility (adjusted $r=0.5(P<0.001)$ and $r=0.43(P<0.001)$, respectively) and pre- and post-cryopreservation viability (adjusted $r=0.35 \quad(P<0.005)$ and $r=0.46 \quad(P<0.001)$, respectively) were maintained after adjustment. As expected on the basis of our previous study (Muratori et al. 2008b), $\mathrm{PI}^{\text {dimmer }}$ SDF was negatively correlated with pre- and postcryopreservation total and progressive motility and viability in both group of patients (Table 2). There were no relationships between total SDF and pre- and post-cryopreservation sperm motility or viability in cancer patients, although there was a negative relationship between total SDF and pre- and postcryopreservation motility in non-cancer patients, likely driven by the high percentage of PI ${ }^{\text {dimmer }}$ SDF of subfertile patients.

\section{Discussion}

The present study clearly demonstrates that, regardless of the type of cancer (haematological or testicular) and of post-thaw motility and viability, $\mathrm{PI}^{\text {brighter }}$ SDF is significantly higher in cryopreserved spermatozoa from cancer patients compared with men cryopreserving samples because of subfertility or autoimmune pathologies. Of importance, the positive correlation between SDF in the PI ${ }^{\text {brighter }}$ population and post-thaw motility in cancer patients strongly suggests that spermatozoa from these patients with damaged DNA may be motile. In this scenario, there is an increase in the probability that a motile spermatozoon with fragmented DNA is involved in oocyte fertilisation when cryopreserved spermatozoa from cancer patients are used for ART.

$\mathrm{PI}^{\text {brighter }} \mathrm{SDF}$ is the only post-thaw sperm parameter of all those evaluated in the present study that discriminates between sperm samples of patients cryopreserving samples because of cancer and those cryopreserving samples because of non-cancer pathologies. Indeed, in agreement with previous studies (Degl'Innocenti et al. 2013), we found that recovery of sperm motility and viability after thawing in cancer groups did not differ from that in patients cryopreserving samples because of autoimmune pathologies (with the exception of seminoma patients), and was even higher compared with subfertile patients. Similarly, SDF in the total sperm population only discriminates between cancer and autoimmune pathology patients, and not between men with cancer and subfertile men, likely because of the higher levels of $\mathrm{PI}^{\mathrm{dimmer}} \mathrm{SDF}$ in the latter. As mentioned above, the PI ${ }^{\text {dimmer }}$ sperm population is formed by unviable spermatozoa (Marchiani et al. 2011; Muratori et al. 2015) with no chance of participating in the fertilisation process. Overall, the present study reveals that, among patients cryopreserving samples for different types of pathology, post-cryopreservation SDF is increased in the clinically relevant sperm population ( $\mathrm{PI}^{\text {brighter }}$ ) only in cancer patients.

Higher SDF levels in post-cryopreserved spermatozoa from cancer patients may be related to agents released from cancer cells as part of the paraneoplastic syndrome (Agarwal and Allamaneni 2005), which may induce apoptosis in the testis or during sperm transit in the male genital tract or, if present in seminal plasma, after ejaculation. Indeed, apoptotic insults are known to cause SDF (Tamburrino et al. 2012; Muratori et al. 2015). However, as mentioned above, whether the occurrence of cancer is per se a factor favouring SDF is questioned, because variable results, depending on the type of tumour and on the method used to evaluate the parameter, have been reported (O'Flaherty et al. 2008; Meseguer et al. 2008; Ribeiro et al. 2008; Ståhl et al. 2009; McDowell et al. 2013). As such, we cannot exclude the possibility that the high postcryopreservation levels of SDF in cancer patients (Edelstein et al. 2008; Meseguer et al. 2008; present study) reflect damage due to the continuous presence of an altered seminal plasma during the cryopreservation procedure (including the thawing procedure, which lasts several minutes).

It has been demonstrated that SDF levels increase with age (Moskovtsev et al. 2006; Wyrobek et al. 2006; Schmid et al. 2007). In the present study, the age at the moment of cryopreservation was lower in cancer patients compared with noncancer patients, thus excluding the possibility that higher SDF levels in the former group were due to an age effect. However, we cannot exclude the possibility that there was an age effect contributing to the higher total (and $\mathrm{PI}^{\text {dimmer }}$ ) SDF levels in subfertile men, who were older compared with all the other patient groups at the time of cryopreservation.

The positive correlation between PI ${ }^{\text {brighter }}$ SDF and both preand post-cryopreservation sperm motility only in cancer patients is further evidence that the presence of malignancies affects sperm DNA integrity, but not motility and viability. Interestingly, this correlation was not affected by the type of cancer present, as indicated by the persistence of significant correlations after adjustment for cancer types. The lack of SDF values before cryopreservation (discussed below) did not allow us to investigate whether motile spermatozoa from cancer patients are more susceptible to cryodamage or whether the damage in motile spermatozoa was already present at the time of cryopreservation.

One drawback of the present study, and also the studies of Meseguer et al. (2008) and Edelstein et al. (2008), is the lack of SDF data at the time of cryopreservation. Although we planned to analyse SDF at the time of cryopreservation, this was not possible for ethical reasons: we could not ask patients to provide a further semen sample for research purposes either because of their psychological condition or because, in some cases, another semen collection was needed to ensure a sufficient number of spermatozoa for cryopreservation. The lack of precryopreservation SDF values did not allow us to verify whether the higher SDF in cancer patients is due to increased SDF at the moment of cryopreservation or to a higher susceptibility of these samples to cryodamage. In the latter case, we may hypothesise that the persistence of harmful substances present in the seminal plasma of cancer patients may have played a role. 
The findings of the present study may contribute to explaining the lower pregnancy rate when cryopreserved semen from cancer patients is used in IVF cycles (Kelleher et al. 2001; Lass et al. 2001; Ragni et al. 2003; Agarwal et al. 2004; Chung et al. 2004; van Casteren et al. 2008). Studies in animals and men demonstrate that DNA-fragmented spermatozoa can fertilise an oocyte and produce viable embryos (Ahmadi and $\mathrm{Ng}$ 1999; Fatehi et al. 2006; Pérez-Cerezales et al. 2010; Yamauchi et al. 2012). However, such embryos may fail to develop to blastocysts (Fatehi et al. 2006) or, if transferred to a uterus, fail to implant or, in case of implantation, result in higher miscarriage rates (Zini et al. 2008; Robinson et al. 2012). Even though it has been demonstrated that the oocyte and embryo retain the ability to repair DNA damage brought by the paternal genome (Ménézo et al. 2010), this ability depends on the extent and type of damage (Derijck et al. 2008). Of interest, a recent case report (Herrero et al. 2013) demonstrated that sorting spermatozoa after magnetic elimination of apoptotic (annexin V-positive) spermatozoa from frozen semen samples of a man who had cryopreserved samples because of cancer decreased SDF and led to a successful live birth with ICSI after two previous unsuccessful attempts with unselected spermatozoa. Because we have recently demonstrated that DNA-fragmented PI ${ }^{\text {brighter }}$ spermatozoa show signs of apoptosis (Muratori et al. 2015), eliminating apoptotic spermatozoa following cryopreservation could help decrease PI ${ }^{\text {brighter }}$ SDF levels.

In addition to a lack of pre-cryopreservation SDF data, another limitation of the present study concerns control subjects. Although ideal control subjects should be healthy normozoospermic men, the control group in the present study consisted of men who were cryopreserving samples because of oligozoospermia or for autoimmune pathologies, which may affect SDF. However, we recently evaluated PI ${ }^{\text {brighter }}$ SDF levels in cryopreserved spermatozoa from normozoospermic men as part of a different project and found similar values to those observed in the two non-cancer groups in the present study (Marchiani et al. 2014b).

\section{Conclusions}

In conclusion, the present study demonstrates the occurrence of higher levels of $\mathrm{PI}^{\text {brighter }}$ SDF in cryopreserved spermatozoa from cancer patients compared with non-cancer patients. The positive relationship between $\mathrm{PI}^{\text {brighter }} \mathrm{SDF}$ and sperm motility in cancer patients indicates that, after thawing, motile spermatozoa involved in the fertilisation process during IVF may carry DNA damage. Recent meta-analyses indicate that SDF affects the results of ART (Zini et al. 2008; Robinson et al. 2012), particularly affecting the percentage of miscarriages. In light of this, the results of the present study could be useful to complement genetic counselling during the routine management of cancer patients in order to preserve their fertility.

\section{Acknowledgements}

This work was supported by grants from the Italian Ministry of University and Scientific Research (project FIRB to SM; and PRIN to EB) and Regione Toscana (to GF). The authors thank Dr E. Filimberti and M. G. Fino for helpful advice.

\section{References}

Agarwal, A., and Allamaneni, S. S. (2005). Disruption of spermatogenesis by the cancer disease process. J. Natl Cancer Inst. Monogr. 2005, 9-12. doi:10.1093/JNCIMONOGRAPHS/LGI005

Agarwal, A., Ranganathan, P., Kattal, N., Pasqualotto, F., Hallak, J., Khayal, S., and Mascha, E. (2004). Fertility after cancer: a prospective review of assisted reproductive outcome with banked semen specimens. Fertil. Steril. 81, 342-348. doi:10.1016/J.FERTNSTERT.2003.07.021

Ahmadi, A., and Ng, S. C. (1999). Fertilizing ability of DNA-damaged spermatozoa. J. Exp. Zool. 284, 696-704. doi:10.1002/(SICI)1097-010X (19991101)284:6<696::AID-JEZ11>3.0.CO;2-E

Bizet, P., Saias-Magnan, J., Jouve, E., Grillo, J. M., Karsenty, G., MetzlerGuillemain, C., and Perrin, J. (2012). Sperm cryopreservation before cancer treatment: a 15-year monocentric experience. Reprod. Biomed. Online 24, 321-330. doi:10.1016/J.RBMO.2011.11.015

Botchan, A., Karpol, S., Lehavi, O., Paz, G., Kleiman, S. E., Yogev, L., Yavetz, H., and Hauser, R. (2013). Preservation of sperm of cancer patients: extent of use and pregnancy outcome in a tertiary infertility center. Asian J. Androl. 15, 382-386. doi:10.1038/AJA.2013.3

Bujan, L., Walschaerts, M., Brugnon, F., Daudin, M., Berthaut, I., Auger, J., Saias, J., Szerman, E., Moinard, N., Rives, N., and Hennebicq, S. (2014). Impact of lymphoma treatments on spermatogenesis and sperm deoxyribonucleic acid: a multicenter prospective study from the CECOS network. Fertil. Steril. 102, 667-674.e3. doi:10.1016/J.FERTNSTERT. 2014.06.008

Chung, K., Irani, J., Knee, G., Efymow, B., Blasco, L., and Patrizio, P. (2004). Sperm cryopreservation for male patients with cancer: an epidemiological analysis at the University of Pennsylvania. Eur. J. Obstet. Gynecol. Reprod. Biol. 113, S7-S11. doi:10.1016/ J.EJOGRB.2003.11.024

Clarke, G. N., Bourne, H., Hill, P., Johnston, W. I., Speirs, A., McBain, J. C., and Baker, H. W. (1997). Artificial insemination and in-vitro fertilization using donor spermatozoa: a report on 15 years of experience. Hum. Reprod. 12, 722-726. doi:10.1093/HUMREP/12.4.722

Degl'Innocenti, S., Filimberti, E., Magini, A., Krausz, C., Lombardi, G., Fino, M. G., Rastrelli, G., Maggi, M., and Baldi, E. (2013). Semen cryopreservation for men banking for oligospermia, cancers, and other pathologies: prediction of post-thaw outcome using basal semen quality. Fertil. Steril. 100, 1555-1563.e3. doi:10.1016/J.FERTNSTERT.2013. 08.005

Derijck, A., van der Heijden, G., Giele, M., Philippens, M., and de Boer, P. (2008). DNA double-strand break repair in parental chromatin of mouse zygotes, the first cell cycle as an origin of de novo mutation. Hum. Mol. Genet. 17, 1922-1937. doi:10.1093/HMG/DDN090

Domínguez-Fandos, D., Camejo, M. I., Ballescà, J. L., and Oliva, R. (2007). Human sperm DNA fragmentation: correlation of TUNEL results as assessed by flow cytometry and optical microscopy. Cytometry $A \mathbf{7 1 A}$, 1011-1018. doi:10.1002/CYTO.A.20484

Edelstein, A., Yavetz, H., Kleiman, S. E., Botchan, A., Hauser, R., Paz, G., and Yogev, L. (2008). Deoxyribonucleic acid-damaged sperm in cryopreserved-thawed specimens from cancer patients and healthy men. Fertil. Steril. 90, 205-208. doi:10.1016/J.FERTNSTERT.2007.05.060

Fatehi, A. N., Bevers, M. M., Schoevers, E., Roelen, B. A., Colenbrander, B., and Gadella, B. M. (2006). DNA damage in bovine sperm does not block fertilization and early embryonic development but induces apoptosis after the first cleavages. J. Androl. 27, 176-188. doi:10.2164/ JANDROL.04152

Gandini, L., Lombardo, F., Lenzi, A., Spanò, M., and Dondero, F. (2006) Cryopreservation and sperm DNA integrity. Cell Tissue Bank. 7, 91-98. doi: 10.1007/S10561-005-0275-8

Hallak, J., Kolletis, P. N., and Sekhon, V. S. (1999). Sperm cryopreservation in patients with testicular cancer. Urology 54, 894-899. doi:10.1016 S0090-4295(99)00267-8 
Henkel, R., Hoogendijk, C. F., Bouic, P. J., and Kruger, T. F. (2010). TUNEL assay and SCSA determine different aspects of sperm DNA damage. Andrologia 42, 305-313. doi:10.1111/J.1439-0272.2009.01002.X

Herrero, M. B., Delbes, G., Chung, J. T., Son, W. Y., Holzer, H., Buckett, W., and Chan, P. (2013). Case report: the use of annexin V coupled with magnetic activated cell sorting in cryopreserved spermatozoa from a male cancer survivor: healthy twin newborns after two previous ICSI failures. J. Assist. Reprod. Genet. 30, 1415-1419. doi:10.1007/S10815013-0086-Y

Kelleher, S., Wishart, S. M., Liu, P. Y., Turner, L., Di Pierro, I., Conway, A. J., and Handelsman, D. J. (2001). Long-term outcomes of elective human sperm cryostorage. Hum. Reprod. 16, 2632-2639. doi:10.1093/ HUMREP/16.12.2632

Lass, A., Akagbosu, F., and Brinsden, P. (2001). Sperm banking and assisted reproduction treatment for couples following cancer treatment of the male partner. Hum. Reprod. Update 7, 370-377. doi:10.1093/ HUMUPD/7.4.370

Marchiani, S., Tamburrino, L., Maoggi, A., Vannelli, G. B., Forti, G., Baldi, E., and Muratori, M. (2007). Characterization of M540 bodies in human semen: evidence that they are apoptotic bodies. Mol. Hum. Reprod. 13, 621-631. doi:10.1093/MOLEHR/GAM046

Marchiani, S., Tamburrino, L., Giuliano, L., Nosi, D., Sarli, V., Gandini, L., Piomboni, P., Belmonte, G., Forti, G., Baldi, E., and Muratori, M. (2011). Sumol-ylation of human spermatozoa and its relationship with semen quality. Int. J. Androl. 34, 581-593. doi:10.1111/J.1365-2605. 2010.01118.X

Marchiani, S., Tamburrino, L., Olivito, B., Betti, L., Azzari, C., Forti, G., Baldi, E., and Muratori, M. (2014a). Characterization and sorting of flow cytometric populations in human semen. Andrology 2, 394-401. doi:10.1111/J.2047-2927.2014.00208.X

Marchiani, S., Tamburrino, L., Ricci, B., Nosi, D., Cambi, M., Piomboni, P., Belmonte, G., Forti, G., Muratori, M., and Baldi, E. (2014b). SUMO1 in human sperm: new targets, role in motility and morphology and relationship with DNA damage. Reproduction 148, 453-467. doi:10.1530/REP-14-0173

McDowell, S., Harrison, K., Kroon, B., Ford, E., and Yazdani, A. (2013). Sperm DNA fragmentation in men with malignancy. Fertil. Steril. 99, 1862-1866. doi:10.1016/J.FERTNSTERT.2013.02.015

Ménézo, Y., Dale, B., and Cohen, M. (2010). DNA damage and repair in human oocytes and embryos: a review. Zygote 18, 357-365. doi:10.1017/S0967199410000286

Meseguer, M., Santiso, R., Garrido, N., and Fernandez, J. L. (2008). The effect of cancer on sperm DNA fragmentation as measured by the sperm chromatin dispersion test. Fertil. Steril. 90, 225-227. doi:10.1016/ J.FERTNSTERT.2007.06.026

Moskovtsev, S. I., Willis, J., and Mullen, J. B. (2006). Age-related decline in sperm deoxyribonucleic acid integrity in patients evaluated for male infertility. Fertil. Steril. 85, 496-499. doi:10.1016/J.FERTNSTERT. 2005.05.075

Muratori, M., Forti, G., and Baldi, E. (2008a). Comparing flow cytometry and fluorescence microscopy for analyzing human sperm DNA fragmentation by TUNEL labeling. Cytometry $A$ 73A, 785-787. doi:10.1002/ CYTO.A.20615

Muratori, M., Marchiani, S., Tamburrino, L., Tocci, V., Failli, P., Forti, G., and Baldi, E. (2008b). Nuclear staining identifies two populations of human sperm with different DNA fragmentation extent and relationship with semen parameters. Hum. Reprod. 23, 1035-1043. doi:10.1093/ HUMREP/DEN058

Muratori, M., Tamburrino, L., Tocci, V., Costantino, A., Marchiani, S., Giachini, C., Laface, I., Krausz, C., Meriggiola, M. C., Forti, G., and Baldi, E. (2010). Small variations in crucial steps of TUNEL assay coupled to flow cytometry greatly affect measures of sperm DNA fragmentation. J. Androl. 31, 336-345. doi:10.2164/JANDROL. 109.008508

Muratori, M., Tamburrino, L., Marchiani, S., Cambi, M., Olivito, B., Azzari, C., Forti, G., and Baldi, E. (2015). Investigation on the origin of sperm DNA fragmentation: role of apoptosis, immaturity and oxidative stress. Mol. Med. 21, 109-122. doi:10.2119/MOLMED.2014.00158

Nordqvist, S., Sydsjö, G., Lampic, C., Åkerud, H., Elenis, E., and Skoog Svanberg, A. (2014). Sexual orientation of women does not affect outcome of fertility treatment with donated sperm. Hum. Reprod. 29 , 704-711. doi:10.1093/HUMREP/DET445

O'Flaherty, C., Vaisheva, F., Hales, B. F., Chan, P., and Robaire, B. (2008). Characterization of sperm chromatin quality in testicular cancer and Hodgkin's lymphoma patients prior to chemotherapy. Hum. Reprod. 23, 1044-1052. doi:10.1093/HUMREP/DEN081

Pérez-Cerezales, S., Martínez-Páramo, S., Beirão, J., and Herráez, M. P. (2010). Fertilization capacity with rainbow trout DNA-damaged sperm and embryo developmental success. Reproduction 139, 989-997. doi:10.1530/REP-10-0037

Ping, P., Gu, B. H., Li, P., Huang, Y. R., and Li, Z. (2014). Fertility outcome of patients with testicular tumor: before and after treatment. Asian J. Androl. 16, 107-111. doi:10.4103/1008-682X.122194

Ragni, G., Somigliana, E., Restelli, L., Salvi, R., Arnoldi, M., and Paffoni, A. (2003). Sperm banking and rate of assisted reproduction treatment: insights from a 15-year cryopreservation program for male cancer patients. Cancer 97, 1624-1629. doi:10.1002/CNCR.11229

Ribas-Maynou, J., Fernández-Encinas, A., García-Peiró, A., Prada, E., Abad, C., Amengual, M. J., Navarro, J., and Benet, J. (2014). Human semen cryopreservation: a sperm DNA fragmentation study with alkaline and neutral Comet assay. Andrology 2, 83-87. doi:10.1111/J.2047-2927. 2013.00158.X

Ribeiro, T. M., Bertolla, R. P., Spaine, D. M., Fraietta, R., Ortiz, V., and Cedenho, A. P. (2008). Sperm nuclear apoptotic DNA fragmentation in men with testicular cancer. Fertil. Steril. 90, 1782-1786. doi:10.1016/ J.FERTNSTERT.2007.08.012

Robinson, L., Gallos, I. D., Conner, S. J., Rajkhowa, M., Miller, D., Lewis, S., Kirkman-Brown, J., and Coomarasamy, A. (2012). The effect of sperm DNA fragmentation on miscarriage rates: a systematic review and meta-analysis. Hum. Reprod. 27, 2908-2917. doi:10.1093/HUMREP/ DES261

Rofeim, O., and Gilbert, B. R. (2004). Normal semen parameters in cancer patients presenting or cryopreservation before gonadotoxic therapy. Fertil. Steril. 82, 505-506. doi:10.1016/J.FERTNSTERT.2003.12.045

Said, T. M., Tellez, S., Evenson, D. P., and Del Valle, A. P. (2009). Assessment of sperm quality, DNA integrity and cryopreservation protocols in men diagnosed with testicular and systemic malignancies. Andrologia 41, 377-382. doi:10.1111/J.1439-0272.2009.00941.X

Schmid, T. E., Eskenazi, B., Baumgartner, A., Marchetti, F., Young, S., Weldon, R., Anderson, D., and Wyrobek, A. J. (2007). The effects of male age on sperm DNA damage in healthy non-smokers. Hum. Reprod. 22, 180-187. doi:10.1093/HUMREP/DEL338

Smit, M., van Casteren, N. J., Wildhagen, M. F., Romijn, J. C., and Dohle, G. R. (2010). Sperm DNA integrity in cancer patients before and after cytotoxic treatment. Hum. Reprod. 25, 1877-1883. doi:10.1093/ HUMREP/DEQ104

Ståhl, O., Eberhard, J., Cavallin-Ståhl, E., Jepson, K., Friberg, B., Tingsmark, C., Spanò, M., and Giwercman, A. (2009). Sperm DNA integrity in cancer patients: the effect of disease and treatment. Int. J. Androl. 32, 695-703. doi:10.1111/J.1365-2605.2008.00933.X

Tamburrino, L., Marchiani, S., Montoya, M., Marino, F. E., Natali, I., Cambi, M., Forti, G., Baldi, E., and Muratori, M. (2012). Mechanisms and clinical correlates of sperm DNA damage. Asian J. Androl. 14, 24-31. doi:10.1038/AJA.2011.59 
Tomlinson, M. J., Pooley, K., Pierce, A., and Hopkisson, J. F. (2010). Sperm donor recruitment within an NHS fertility service since the removal of anonymity. Hum. Fertil. (Camb.) 13, 159-167. doi:10.3109/14647273. 2010.512654

van Casteren, N. J., van Santbrink, E. J. P., van Inzen, W., Romijn, J. C., and Dohle, G. R. (2008). Use rate and assisted reproduction technologies outcome of cryopreserved semen from 629 cancer patients. Fertil. Steril. 90, 2245-2250. doi:10.1016/J.FERTNSTERT.2007.10.055

World Health Organization (WHO). (1999). 'WHO Laboratory Manual for the Examination and Processing of Human Semen.' 4th edn. (Cambridge University Press: Cambridge, UK.)

World Health Organization (WHO). (2010). 'WHO Laboratory Manual for the Examination and Processing of Human Semen.' 5th edn. (WHO Press: Geneva.)

Wyrobek, A. J., Eskenazi, B., Young, S., Arnheim, N., Tiemann-Boege, I., Jabs, E. W., Glaser, R. L., Pearson, F. S., and Evenson, D. (2006)
Advancing age has differential effects on DNA damage, chromatin integrity, gene mutations, and aneuploidies in sperm. Proc. Natl Acad. Sci. USA 103, 9601-9606. doi:10.1073/PNAS.0506468103

Yamauchi, Y., Riel, J. M., and Ward, M. A. (2012). Paternal DNA damage resulting from various sperm treatments persists after fertilization and is similar prior and after DNA replication. J. Androl. 33, 229-238. doi:10.2164/JANDROL.111.013532

Zini, A., Boman, J. M., Belzile, E., and Ciampi, A. (2008). Sperm DNA damage is associated with an increased risk of pregnancy loss after IVF and ICSI: systematic review and meta-analysis. Hum. Reprod. 23 , 2663-2668. doi:10.1093/HUMREP/DEN321

Zribi, N., Feki Chakroun, N., El Euch, H., Gargouri, J., Bahloul, A., and Ammar Keskes, L. (2010). Effects of cryopreservation on human sperm deoxyribonucleic acid integrity. Fertil. Steril. 93, 159-166. doi:10.1016/ J.FERTNSTERT.2008.09.038 